\title{
Large resonant third-order optical nonlinearity of thin film containing J-like aggregates of a bis[4-( $N$-dibutylamino)phenyl]squarylium dye
}

\author{
ZHONGYU LI ${ }^{\mathrm{a}, \mathrm{b}, \mathrm{c}, *}$, SONG XU ${ }^{\mathrm{a}}$, LIN ZHU ${ }^{\mathrm{c}}$ and KAZUO KASATANI ${ }^{\mathrm{d}}$ \\ ${ }^{a}$ School of Petrochemical Engineering, Changzhou University, Changzhou 213164, PR China \\ ${ }^{\mathrm{b}}$ Changzhou Expansion New Stuff Technology Limited Company, Changzhou 213122, PR China \\ ${ }^{c}$ Department of Materials and Chemical Engineering, Jilin Institute of Chemical Technology, Jilin 132022, PR China \\ ${ }^{\mathrm{d}}$ Department of Advanced Materials Science and Engineering, Faculty of Engineering, Yamaguchi University, Tokiwadai, \\ Ube 755-8611, Japan
}

MS received 8 November 2011; revised 16 February 2012

\begin{abstract}
The third-order optical nonlinearity and response of thin film containing J-like aggregates of a bis[4( $N$-dibutylamino)phenyl]squarylium dye were measured by degenerate four-wave mixing (DFWM) technique under resonant conditions. The temporal profile of DFWM signal was obtained with a time resolution of 0.3 ps (FWHM), and was found to consist of at least two components, i.e. the coherent instantaneous nonlinear response (electronic response) and the slow response due to the excited state population grating. The effective $\chi^{(3)}$ value of thin squarylium dye film was evaluated to be as high as $1.1 \times 10^{-7} \mathrm{esu}$, and the figure of merit of third-order nonlinearity $F\left(F=\chi^{(3)} / \alpha\right)$, was calculated to be about $2.1 \times 10^{-13}$ esu cm.
\end{abstract}

Keywords. Degenerate four-wave mixing; J-like aggregates; third-order nonlinear susceptibility; squarylium dye.

\section{Introduction}

Nonlinear optical films with large optical nonlinearity and ultrafast response are required as promising candidates for future integrated photonics applications, such as alloptical switching, dynamic holography, data storage, optical communication, etc (Norwood and Sounik 1992; Nalwa 1993; Liu et al 2008; Hou et al 2010; Chen et al 2011; Sabari Girisun et al 2011). Among optical materials, aggregates of organic dyes are ordered molecular assemblies with mesoscopic size and are different from molecular crystals due to their specific optical properties. Cyanine dyes can readily form large aggregated structures that consist of several tens to hundreds of molecular units either by electrostatic or van der Waals interaction. This phenomenon has been observed as early as in the 1930s by Jelley (1936) and Scheibe (1937). The corresponding type of molecular aggregates is referred to as J-aggregates. The most characteristic feature of J-aggregates is that they exhibit a narrow peak (J-band) red-shifted in the absorption spectrum with respect to the monomer absorption.

Because of the resonant dipole-dipole interaction between adjacent molecules and the translational symmetry of the system, absorption of a photon produces a coherent delocalized excitation or exciton in the J-aggregates. An exciton involves a collective oscillation of $N$ adjacent dipoles in a chain or in a lattice of molecules, leading to an $N$-fold enhancement of

*Author for correspondence (zhongyuli@mail.tsinghua.edu.cn; zhongyuli@cczu.edu.cn) the transition oscillator strength and a corresponding increase in the probability of absorption and emission of light. In the J-aggregates, high oscillator strength value manifests itself as a drastic shortening of the spontaneous radiative decay time (Boer and Wiersma 1990). The lifetime of the exciton can be further reduced by an exciton-exciton annihilation process, resulting in a picosecond or sub-picosecond relaxation rate. On the other hand, high oscillator strength also provides an increased resonant nonlinear susceptibility value (Spano and Mukamel 1989a). In the present study, we found that bis[4( $N$-dibutylamino)phenyl]squarylium dye forms J-like aggregates in thin film, and the third-order nonlinear optical property of J-like aggregates of this squarylium dye was measured by the femtosecond degenerate four-wave mixing (DFWM) technique under resonant conditions.

\section{Experimental}

The bis[4-( $N$-dibutylamino)phenyl]squarylium dye (structural formula as shown in figure 1) was synthesized in accordance with a reported procedure (Sprenger and Ziegenbein 1966). Film of the squarylium dye J-like aggregates was formed on glass substrates by the spin-coating method from a solution of bis[4-( $N$-dibutylamino)phenyl]squarylium dye at a concentration of $1.0 \mathrm{wt} \%$ dissolved in 1,2-dichloroethane. The film has high optical qualities with good optical transparency, excellent homogeneity and optical stability.

Film thickness was determined to be about $100 \mathrm{~nm}$ using a surface profiler (ULVAC, Dektak ${ }^{3}$ ). All the absorption 


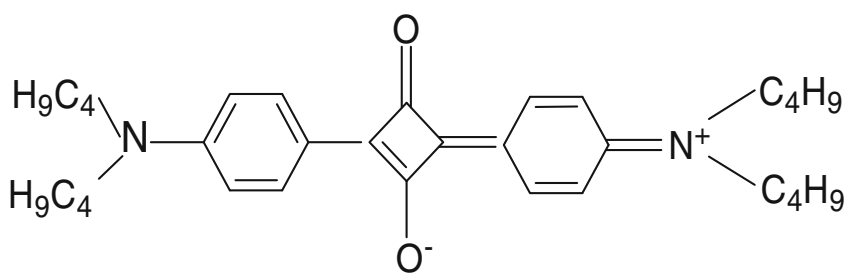

Figure 1. Structural formula of bis[4-( $N$-dibutylamino $)$ phenyl]squarylium dye.

spectra of samples were recorded with a Jasco V-550 spectrophotometer. Time-resolved fluorescence lifetime measurements were carried out using a combination of a Ti:sapphire laser (Spectra Physics, Tsunami) and a streak camera (Hamamatsu, Streak Scope C4334).

The third-order nonlinear optical property of the sample was measured using the forward DFWM technique. The system used in our experimental study of DFWM is the same as that described in previous papers (Li et al 2005a,b). The folded box-CARS type geometry with three linearly polarized beams of the fundamental output of a Ti:sapphire regenerative amplifier laser (Spectra-Physics, Spitfire, $130 \mathrm{fs,}$ $1 \mathrm{kHz}, 1 \mathrm{~W}$ ) was used for DFWM experiment. Two of the three forward beams are used for the generation of a refractive index grating in sample, while the third beam is used for the detection of this grating, i.e. the light-induced change of the refractive index. The wavelength of the laser was adjusted to $760 \mathrm{~nm}$. The laser beam was largely attenuated by ND filters in order to avoid saturation. The energies of beams 1 and 2 were $<100 \mathrm{~nJ} /$ pulse and that of beam 3 was $<10 \mathrm{~nJ} /$ pulse. The intensity of the laser radiation on samples was about $1 \mathrm{GW} / \mathrm{cm}^{2}$. The sample was simultaneously irradiated with beams 1 and 2, and then irradiated with beam 3 after a suitable delay time. The three collinear beams were focused onto the sample by a lens with a $500 \mathrm{~mm}$ focal length. Beam 4 , the produced DFWM signal, was detected by a CCD after passing through a monochromator. The output of CCD was acquired using a personal computer. The time resolution of the system was ca. $0 \cdot 3$ ps (FWHM). The intensity of DFWM signal due to the solvent and quartz cuvette was completely negligible. The experimental uncertainty was estimated to be within $\pm 20 \%$.

The effective third-order nonlinear susceptibility, $\chi^{(3)}$, was calculated by comparison with the signal of a reference sample, $\mathrm{CCl}_{4}\left(\chi^{(3)}=4.01 \times 10^{-14} \mathrm{esu}\right)($ Kamada et al 1996) measured under the same conditions, and was corrected for the absorption losses according to the following relationship

$$
\begin{aligned}
\chi_{\text {sample }}^{(3)}= & \left(n_{\text {sample }} / n_{\text {ref }}\right)^{2}\left(I_{\text {sample }} / I_{\text {ref }}\right)^{1 / 2}\left(L_{\text {ref }} / L_{\text {sample }}\right) \\
& \times \alpha L_{\text {sample }} \exp \left(\alpha L_{\text {sample }} / 2\right) \\
& \times\left[1-\exp \left(-\alpha L_{\text {sample }}\right)\right]^{-1} \chi_{\text {ref }}^{(3)}
\end{aligned}
$$

where $I$ is the DFWM signal intensity, $\alpha$ the linear absorption coefficient, $n$ the refractive index and $L$ the interaction

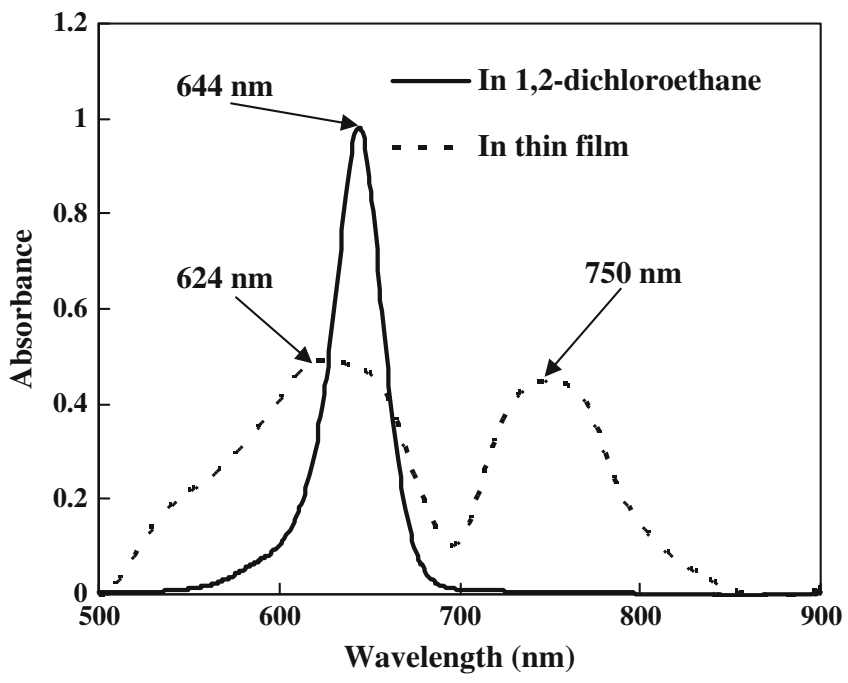

Figure 2. Absorption spectra of bis[4-( $N$-dibutylamino)phenyl]squarylium dye in dilute 1,2-dichloroethane solution (solid line) and in a thin film (dotted line).

length. The subscript 'ref' refers to the reference. Instead of using $\mathrm{CS}_{2}$, we used $\mathrm{CCl}_{4}$ as a reference. The reason is that the molecular anisotropy of $\mathrm{CS}_{2}$ makes it undergo molecular reorientation under the action of laser pulses and give rise to orientation nonlinearity. The rise and fall times for this orientational contribution to DFWM signal are 200 and $>600$ fs, respectively (Ruhman et al 1987; McMorrow et al 1988). Therefore, it is not appropriate in femtosecond regime to use the known effective $\chi^{(3)}$ value of $\mathrm{CS}_{2}$ as reference to evaluate the effective $\chi^{(3)}$ value of sample. On the other hand, isotropic structure of $\mathrm{CCl}_{4}$ and its instantaneous response makes $\mathrm{CCl}_{4}$ an excellent reference material for femtosecond DFWM.

\section{Results and discussion}

Figure 2 shows absorption spectra of bis[4-( $N$-dibutylamino) phenyl]squarylium dye in dilute 1,2-dichloroethane solution (solid line) and in a thin film (dotted line). The squarylium dye in 1,2-dichloroethane dilute solution exhibits intense absorption in the visible region with absorption maximum at $644 \mathrm{~nm}$. The large extinction coefficient and narrow bandwidth are consistent with the donor-acceptor-donor (D-A-D) electron transfer character of the $S_{0} \rightarrow S_{1}$ absorption (Bigelow and Freund 1986). It can be seen that the absorption spectrum of the squarylium dye thin film is much broader, which has a longer maximum wavelength at $750 \mathrm{~nm}$ and a blue-shift absorption band at $624 \mathrm{~nm}$ compared to that of 1,2-dichloroethane solution. Drobizhev et al (2000) attributed H-band (H-aggregates) to the upper Davydov component and J-band (J-aggregates) to lower Davydov component. The absorption of the J-aggregates was influenced by size of the aggregate, interfacial distance between the neighbouring dyes and slipping angle of the stacked dyes (Miyata et al 1993; 


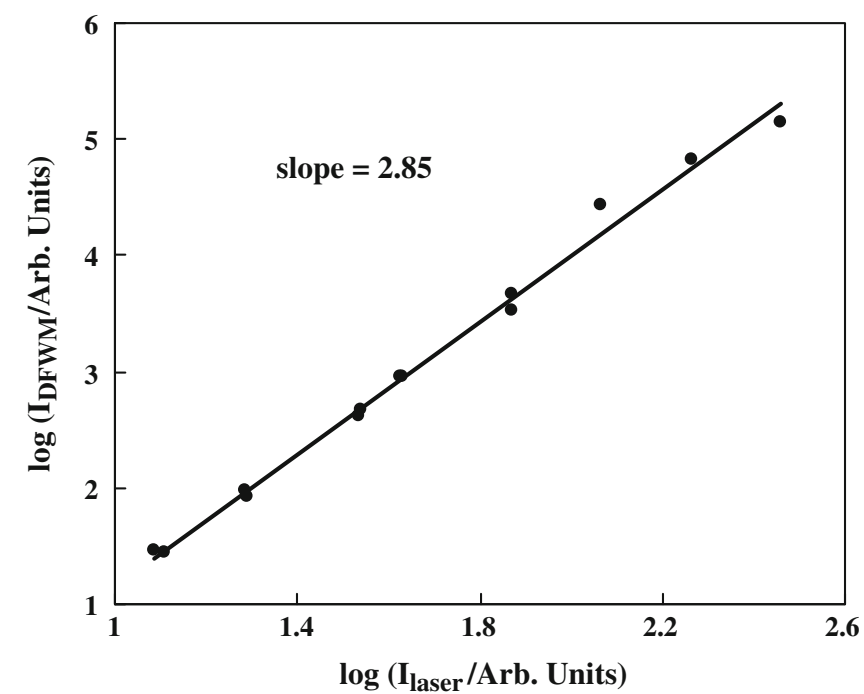

Figure 3. Dependence of DFWM signal intensity of J-like aggregates of bis[4-( $N$-dibutylamino)phenyl]squarylium dye on input laser power.

Sayama et al 2002). The static and dynamic disorder (a wide distribution of different arrangements) of the dye molecules on the glass substrate probably caused broad absorption spectra. We refer to the aggregates showing a broad absorption at a longer wavelength region as J-like aggregates mentioned below. Therefore, the appeared red-shift and broad band can be attributed to the formation of J-like aggregates of bis[4-( $N$-dibutylamino)phenyl]squarylium dye molecules.

Figure 3 shows a log-log plot of DFWM signal intensity against pump beam intensity for the thin film containing squarylium dye J-like aggregates. The plot has a slope at nearly around 3 indicating a third-order nature of the process. This also shows that there is no saturation of optical nonlinearity under the measured conditions. The temporal profiles of DFWM signal were measured as a function of delay time of beam 3. The result is shown in figure 4. Each temporal profile has at least two components, a rapidly decaying component and a slow component. The fast component is primarily determined by the laser pulse, and can be attributed to the contribution from the electronic response. The slow component appears to be associated with the excited state population grating.

To analyse the temporal profile of a DFWM signal of bis[4-( $N$-dibutylamino)phenyl]squarylium dye film, it was simulated. The observed DFWM temporal profile, $I(t)$ is assumed to be expressed by the following convolution integral

$$
I(t)=\int_{0}^{t} L\left(t^{\prime}\right) R\left(t-t^{\prime}\right) \mathrm{d} t^{\prime},
$$

where $L(t)$ is the temporal profile of the laser and $R(t)$ the DFWM response of sample irradiated by a laser with an

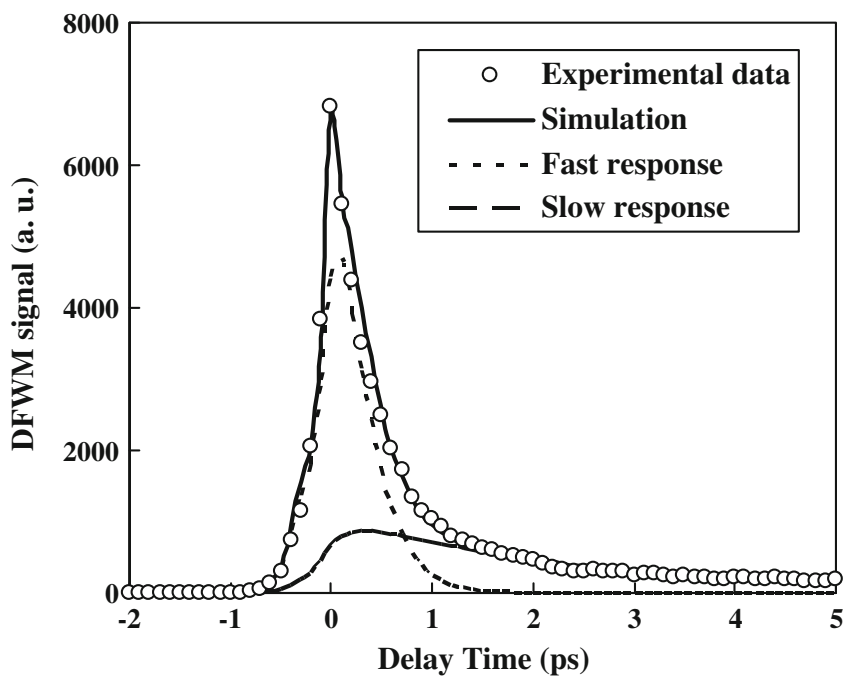

Figure 4. Simulation for a temporal profile of DFWM signal of squarylium dye film by assuming Förster type energy transfer; open circles, solid line, dotted line and broken line represent experimental data, simulation, fast component and slow component, respectively.

ideal delta function shape pulse. $R(t)$ is assumed to be a biexponential function

$$
R(t)=\alpha_{1} \exp \left(-t / \tau_{1}\right)+\alpha_{2} \exp \left(-t / \tau_{2}\right) .
$$

The slow response of a DFWM signal of the squarylium dye film was not exponential. The excited monomer will be quenched by dimer or aggregates. Förster's (1949) theory states that the temporal behaviour of the concentration of excited donor molecules quenched by energy acceptor molecules randomly distributed in a three-dimensional space can be expressed by the following equation

$$
\rho(t)=\exp \left[-t / \tau_{\mathrm{D}}-\gamma_{\mathrm{A}}\left(t / \tau_{\mathrm{D}}\right)^{1 / 2}\right],
$$

where $\tau_{\mathrm{D}}$ is the lifetime of the donor without an acceptor and $\gamma_{\mathrm{A}}$ is a constant related to energy transfer rate.

DFWM signal intensity of a population grating is proportional to the square of the concentration of excited molecules. Therefore, $R(t)$ for the squarylium dye J-like aggregates film can be assumed to be the sum of an exponential function (electronic component) and a slow response (population grating) and expressed as follows

$$
\begin{aligned}
R(t)= & \alpha_{1} \exp \left(-t / \tau_{1}\right)+\alpha_{2} \rho(t)^{2} \\
= & \alpha_{1} \exp \left(-t / \tau_{1}\right) \\
& +\alpha_{2} \exp \left[-2 t / \tau_{\mathrm{D}}-2 \gamma_{\mathrm{A}}\left(t / \tau_{\mathrm{D}}\right)^{1 / 2}\right] .
\end{aligned}
$$

All the parameters in (4) and (5), the time difference between the experimental and simulated DFWM temporal profile, $\Delta t$, were determined using a nonlinear, least-squares iterative convolution method based on the Marquardt algorithm (Marquardt 1963; O'Connor et al 1979). DFWM temporal profile of a glass plate is used as $L(t)$. 
Figure 4 shows simulation of temporal profiles for DFWM signal of thin film containing J-like aggregates of the squarylium dye (solid line), which were simulated in accordance with above referred method by a biexponential function and assuming Förster type energy transfer. It was found that the simulated curve reproduced the experimental values very well. The observed decay of DFWM signal consists of at least two components, i.e. fast component induced by the contribution from the coherent instantaneous nonlinear response (electronic response) and slow component due to the contribution from the population grating of excited state. The fast component has a decay time constant shorter than $0.06 \mathrm{ps}$, which is primarily determined by the laser pulse, and the slow component has a decay time constant of ca. 7.6 ps. Based on simulation, percentage of the slow component is about $23 \%$. Therefore, the contribution from the fast component becomes dominant for the DFWM signal of the squarylium dye film because of aggregation effect on DFWM response.

The time-resolved fluorescence of $\operatorname{bis}[4-(\mathrm{N}-$ dibutylamino)phenyl]squarylium dye thin film is shown in figure 5. It was found that the fluorescence lifetime of the squarylium dye in film is very short and the fluorescence intensity is very weak, which indicates that nonradiative processes are dominant in squarylium dye thin film. Sakakibara et al (2001) reported that solid films of magnesium, chloroaluminum, and metal-free phthalocyanines have much smaller fluorescence quantum yields than the corresponding monomers due to large intermolecular interactions. Within nonradiative decay processes, exciton coupling and energy transfer between squarylium dye molecules probably account for the fast decay of the $S_{1}$ state. We considered that, for the squarylium dye film, which primarily consists of J-like aggregates, energy transfer from site to site within the J-like aggregates gives rise to the shortening of the $S_{1}$ state lifetime.

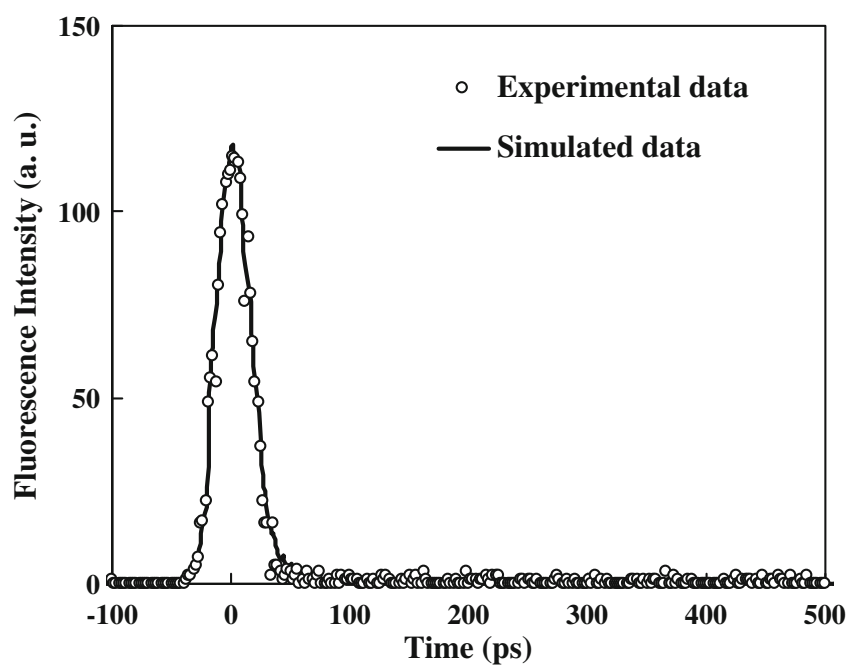

Figure 5. Time-resolved fluorescence of $\operatorname{bis}[4-(N-$ dibutylamino)phenyl]squarylium dye thin film.
We evaluated the electronic component of the third-order nonlinear optical susceptibility, $\chi^{(3)}$, by putting the intensity of the fast component calculated by the corresponding simulation into (1) as $I_{\text {sample }}$. The obtained $\chi^{(3)}$ value is about $1 \cdot 1$ $\times 10^{-7}$ esu at $760 \mathrm{~nm}$. This large third-order nonlinear optical susceptibility can be considered to be induced from the J-like aggregates of squarylium dye formed in the film. Furthermore, the figure of merit of third-order nonlinearity, $F$ $\left(F=\chi^{(3)} / \alpha\right)$, was evaluated to be about $2.1 \times 10^{-13}$ esu $\mathrm{cm}$.

Previous experimental and theoretical works have shown that molecular J-aggregates are self-organized systems that combine a strong third-order optical susceptibility in the exciton absorption band (J-band) with a short response time (Spano and Mukamel 1989b; Kobayashi and Sasaki 1994; Markov et al 2000; Zhou et al 2000; Tatsuura et al 2001). The large optical properties of J-aggregates derive from the delocalization of the Frenkel excitons over many molecules of the aggregate. For perfectly ordered (homogeneous) aggregates the exciton wave functions are entirely delocalized and the delocalization length, $N_{\text {del }}$, is determined by the physical size of aggregates, $N_{\text {del }}=N$. In practice, however, the aggregates contain some amount of disorder, which may severely affect the delocalization behaviour and observed optical properties. Disorder can either be diagonal, meaning a spread in transition frequencies of individual molecules, or off-diagonal, expressing a variation in the intermolecular interactions. Diagonal disorder corresponds to the situation of inhomogeneous broadening due to different molecular surroundings. Off-diagonal or interaction disorder, on the other hand, implies physical irregularity of chain itself. This irregularity can exist in the positions and (or) orientations of the molecules (Fidder et al 1991). As a result, the exciton wave functions localize on a part of the aggregate, with a delocalization length which may be smaller than the physical size of aggregates. A supralinear scaling of the cubic optical nonlinearity, $\chi^{(3)}$, with the exciton delocalization size (so-called size-enhancement) has been theoretically predicted for ordered aggregates as well as for aggregates with static diagonal disorder (Knoester 2003). Markov et al (2003) studied the nanometer films of J-aggregates of pseudoisocyanine (PIC) with various degrees of both static and dynamic disorder, and established the scaling of the nonlinear optical susceptibility per aggregate molecule, $\chi^{(3)} / N$, with the delocalization length as $\chi^{(3)} / N \propto N_{\text {Del }}^{\gamma}$, with $\gamma=$ $2 \cdot 3 \pm 0 \cdot 2$. This power scaling agrees very well with the theoretical prediction of $\gamma=2.36$ for the size-enhancement in one-dimensional aggregates with static diagonal disorder (Knoester 2003). In the present study, considering the J-like aggregates of squarylium dye embedded in a glass environment, the absorption line broadening arising from the interactions with environment should be taken into account. Assuming that the linewidth of the exciton absorption line is dominated by static disorder, static disorder reduces the delocalization of excitation over an aggregate to some amount (exciton delocalization length) of molecular units.

A few resonant $\chi^{(3)}$ values of the $\mathrm{J}$-aggregates of dyes have been reported. For example, the J-aggregates of a 
PIC-Br in LB film $\left(\chi^{(3)}=5 \times 10^{-7}\right.$ esu) (Zhou et al 2000), and Markov et al (2003) reported the nonlinear optical properties of the J-aggregates of PIC iodide with several different $N$-alkyl substituents by the $Z$-scan technique, a giant third-order optical nonlinearity $\left(\chi^{(3)} \approx 10^{-5} \mathrm{esu}\right)$ (Markov et al 2000) was obtained. Because they used a nanosecond pulsed dye laser, we thought that there were contributions to third-order optical nonlinearity from slow responses such as population gratings and thermal gratings. In the present study, we only determined the contribution of electronic response of the $\chi^{(3)}$ for the thin film. Though the absorption band of the J-like aggregates of bis[4( $N$-dibutylamino)phenyl]squarylium dye is a little broader than those of above mentioned J-aggregates, thin film containing bis[4-( $N$-dibutylamino)phenyl]squarylium dye J-like aggregates also show very large third-order optical nonlinearity.

To put in perspective the merits of the studied squarylium dye, we have tried to compare its third-order optical nonlinearity with some of the other molecules reported recently. Kumar et al (2009) measured off-resonant nonlinearities of alkyl phthalocyanines in the range of $\sim 10^{-31}$ esu with fast response times ( $<100 \mathrm{fs}$ ). Our group (Li et al 2007a) presented previously the results on centrosymmetric squaraines possessing large third-order optical nonlinearities studied using femtosecond DFWM technique and achieved $\gamma$ values of $\sim 10^{-31}$ esu. We also reported similar studies on novel diarylethene-phthalocyanine dyads with largest $\gamma$ value for one of the compounds being $\sim 10^{-30}$ esu ( $\mathrm{Li}$ et al 2007b). Prabhakar et al (2008) presented their results of croconate dyes obtained with 100 fs pulses where offresonant $\gamma$ value was found to be $\sim 10^{-32}$ esu. Furthermore, they also reported recently third-order optical nonlinearities of centrosymmetric squaraine derivatives using femtosecond DFWM technique at $800 \mathrm{~nm}$, the $\gamma$ values, though smaller than the squaraine dye having absorption in the red region, are reasonably large and range from -1.2 to $-6 \cdot 9 \times 10^{-33}$ esu (Prabhakar et al 2010). In our present study, thin film containing J-like aggregates of bis[4- $(\mathrm{N}$ dibutylamino)phenyl]squarylium dye shows large third-order nonlinear susceptibility due to resonance enhancement and J-aggregation effect. Though the off-resonant third-order optical nonlinearity of studied squarylium dye in solution has not been measured, we think the $\gamma$ value should also be reasonably large because of significant intramolecular charge transfer (ICT) of squaraine molecules (Dirk et al 1992).

\section{Conclusions}

The magnitude and dynamic response of the third-order optical nonlinearity of J-like aggregates of bis[4- $(\mathrm{N}-$ dibutylamino)phenyl]squarylium dye were measured by femtosecond DFWM technique under resonant condition. Time-resolved DFWM measurement indicates that the J-like aggregates of bis[4-( $N$-dibutylamino)phenyl]squarylium dye shows fast nonlinear optical response and large third-order nonlinear susceptibility. The effective $\chi^{(3)}$ value of the J-like aggregates of bis[4-( $N$-dibutylamino)phenyl] squarylium dye was evaluated to be as high as $1.1 \times 10^{-7} \mathrm{esu}$, and the figure of merit of $\chi^{(3)} / \alpha$ was calculated to be about $2.1 \times 10^{-13}$ esu $\mathrm{cm}$. The present squarylium dye has significant potential for applications in third-order nonlinear optics.

\section{Acknowledgements}

This work was financially supported by the National Natural Science General Foundation of China (Grant Nos, 20572059 and 20502013), the Research Foundation for Talented Scholars of Changzhou University (No. ZMF11020007), and the Natural Science Foundation of Changzhou City (No. CJ20115009).

\section{References}

Bigelow R W and Freund H J 1986 Chem. Phys. 107159

Boer S D and Wiersma D A 1990 Chem. Phys. Lett. 16545

Chen Z, Zhou X, Li Z, Yi J and Zhang F 2011 J. Photochem. Photobiol. A: Chem. 21864

Dirk C W, Cheng L-T and Kuzyk M G 1992 Int. J. Quant. Chem. 4327

Drobizhev M A, Sigel C and Rebane A 2000 J. Lumin. 86107

Fidder H, Knoester J and Wiersma D A 1991 J. Chem. Phys. 95 7880

Förster Th. 1949 Naturforsch. Z. A4 321

Hou Y, Liu Q, Gao C, Qian S and Zhao X 2010 Solid State Commun. 150875

Jelley E E 1936 Nature 1381009

Kamada K, Ueda M, Sakaguchi T, Ohta K and Fukumi T 1996 Chem. Phys. Lett. 263215 (Errata: 1997267 402)

Knoester J 2003 Chem. Phys. Lett. 203371

Kobayashi S and Sasaki F 1994 J. Lumin. 58113

Kumar R S S, Rao S V, Giribabu L and Rao D N 2009 Opt. Mater. 311042

Li Z, Jin Z, Kasatani K, Okamoto H and Takenaka S 2005a Phys. Status Solidi B242 2107

Li Z, Jin Z, Kasatani K, Okamoto H and Takenaka S 2005b Jpn. J. Appl. Phys. 444956

Li Z et al 2007a Chem. Phys. Lett. 441123

Li Z, Chen Z, Xu S, Niu L, Zhang Z, Zhang F and Kasatani K 2007b Chem. Phys. Lett. 447110

Liu Y, Chen Y, Cai L, Wang J, Lin Y and Blau W J 2008 Mater. Chem. Phys. 107189

Markov R V, Plekhanov A I, Shelkovnikov V V and Knoester J 2000 Phys. Status Solidi (b) 221529

Markov R V, Plekhanov A I, Shelkovnikov V V and Knoester J 2003 Microelectron. Eng. 69528

Marquardt D W 1963 J. Soc. Ind. Appl. Math. 11431

McMorrow D, Lotshaw W T and Kenney-Wallace G A 1988 IEEE J. Quant. Elect. 24443

Miyata A, Heard D, Unuma Y and Higashigaki Y 1993 Bull. Chem. Soc. Jap. 66999 
Nalwa H S 1993 Adv. Mater. 5341

Norwood R A and Sounik J R 1992 Appl. Phys. Lett. 60 295

O'Connor D V, Ware W R and Andre J C 1979 J. Phys. Chem. 83 1333

Prabhakar Ch., Yesudas K, Bhanuprakash K, Rao V J, Kumar R S S and Rao D N 2008 J. Phys. Chem. C112 13272

Prabhakar Ch., Bhanuprakash K, Rao V J, Balamuralikrishna M and Rao D N 2010 J. Phys. Chem. C114 6077

Ruhman S, Joly A G, Kohler B, Williams L R and Nelson K A 1987 Rev. Phys. Appl. 221717

Sabari Girisun T C, Dhanuskodi S and Vinitha G 2011 Mater. Chem. Phys. 9129
Sakakibara Y, Bera R N, Mizutani T, Ishida K, Tokumoto M and Tani T 2001 J. Phys. Chem. B105 1547

Sayama K, Tsukagoshi S, Hara K, Ohga Y, Shinpou A, Abe Y, Suga S and Arakawa H 2002 J. Phys. Chem. B106 1363

Scheibe G 1937 Angew. Chem. 50212

Spano F C and Mukamel S 1989a J. Chem. Phys. 91683

Spano F C and Mukamel S 1989b Phys. Rev. A40 5783

Sprenger H E and Ziegenbein W 1966 Angew. Chem. Int. Ed. Engl. 5894

Tatsuura S, Wada O, Tian M, Furuki M, Sato Y, Iwasa I, Pu L S and Kawashima H 2001 Appl. Phys. Lett. 792517

Zhou H S, Watanabe T, Mito A, Asai K, Ishigure K and Honma I 2000 J. Sol-Gel Sci. Technol. 19803 